\title{
BASIC SCIENCE ARTICLE \\ Postnatal outcomes in lambs exposed antenatally and acutely postnatally to fluoxetine
}

\author{
Tuan Anh Nguyen ${ }^{1}$, Timothy Chow ${ }^{2}$, Wayne Riggs ${ }^{2}$ and Dan Rurak ${ }^{1}$
}

\begin{abstract}
BACKGROUND: Approximately $1 / 3$ of newborns exposed antenatally to selective serotonin reuptake inhibitors (SSRIs) exhibit poor neonatal adaptation. Although several potential mechanisms have been proposed, the actual mechanism has not been elucidated. METHODS: We investigated outcomes in neonatal lambs exposed prenatally or postnatally to fluoxetine (FX). Daily FX injections $(50 \mathrm{mg}$ ) were given intravenously (i.v.) to five pregnant ewes via implanted catheters beginning at 131-132 days gestation (term $=$ 147 days) for 2 weeks. In another group, lambs with implanted vascular catheters had sterile water $(n=9)$ or FX $(1 \mathrm{mg} / \mathrm{kg}, n=12)$ injected i.v. on postnatal day (PND) 4.

RESULTS: Prenatal FX-exposed lambs $(n=7)$ were hyperactive during PND 4 to 14 and their heart rate variability (HRV) was significantly lower than in control lambs $(n=7)$ on PND 2. In contrast, arterial pressure, heart rate, electrocardiogram, arterial blood gases, $\mathrm{pH}$, glucose, lactate, cortisol, and sleep-activity cycles were not altered following postnatal FX injection.

CONCLUSION: This abnormal postnatal hyperactivity with antenatal FX exposure may reflect increased maturity in terms of locomotory activity. The results suggest that altered brain development may be involved in the poor neonatal adaptation in human infants exposed to FX in utero.
\end{abstract}

Pediatric Research (2019) 85:1032-1040; https://doi.org/10.1038/s41390-019-0309-8

\section{INTRODUCTION}

Selective serotonin reuptake inhibitors (SSRIs) are widely used for treatment of depression during pregnancy. ${ }^{1}$ However, infants from such pregnancies have higher risks of premature birth, low birth weight, decreased Apgar scores, increased admission to special-care nurseries, reduced postnatal weight gain, and poor neonatal adaptation, ${ }^{2}$ with the latter being most commonly reported. ${ }^{3,4}$ Symptoms include irritability, excessive crying, shivering, tremor, restlessness, poor feeding, respiratory difficulties, sleep disturbances, seizures, dehydration, excessive weight loss, hyperpyrexia, and intubation. These symptoms are transient ${ }^{3}$ and observed in $10-30 \%$ of exposed newborns. ${ }^{5}$ Several mechanisms have been postulated for peptide nucleic acid including an SSRI withdrawal phenomenon in the postnatal period ${ }^{5}$ or drug persistence in the newborn, leading to drug toxicity. ${ }^{3}$ It could also involve a SSRI-elicited alteration in fetal brain development and/or maturation.

In human studies, it is difficult to distinguish between SSRI and maternal psychiatric illness effects. Moreover, the postnatal social environment of a depressed mother could have negative influences on child development. ${ }^{6}$ Since lambs at birth have very similar physiological characteristics to the human, it appears to be a good species to avoid the confounding human factors. Previously, we have reported that fluoxetine (FX) administration to pregnant sheep results in transient decreases in uterine blood flow and fetal vascular $\mathrm{pO}_{2}$, a reduction in fetal REM (rapid eye movement) sleep and increase in quiet sleep and an increase in the fetal prepartum cortisol surge. ${ }^{7}$ However, we collected minimal data on these fetuses following birth. Thus, in the current study, we have investigated lambs postnatally that were exposed to FX during late gestation. In addition to assess whether drug toxicity is involved in poor neonatal adaptation, we assessed the effects of acute neonatal FX administration that resulted in plasma FX concentrations similar to those reported in human newborns following antenatal FX exposure. We hypothesized that while antenatal FX exposure would result in newborn physiologic changes, acute neonatal FX administration would not.

\section{METHODS}

This study was approved by the UBC Committee on Animal Care and conformed to the policies and guidelines of the Canadian Council on Animal Care.

\section{Prenatal FX exposure protocol}

The study involved one group of lambs $(n=7)$ that were exposed to FX antenatally and a control group $(n=7)$ without any perinatal FX exposure. At $131 \pm 1$ day gestation, a heparin-bonded 5.0 French polyurethane catheter (Instech Solomon, Plymouth Meeting, PA, USA) was percutaneously implanted in a maternal jugular vein under local analgesia in five pregnant ewes. It was secured to the skin by a 2-0 Nylon suture. The external catheter opening was closed with a sterile metal pin and kept in a small zip lock bag in a bandage around the neck. The catheters were flushed daily with heparinized saline.

Beginning one day after catheter insertion, the pregnant ewes were given daily injections of $50 \mathrm{mg} F X$ at 10:00-11:00 a.m. for 14 days or until the ewes gave birth, whichever came earlier. Signs of imminent parturition in the ewe and birth were observed by digital video recorder (DVR). The lambs' weight was measured.

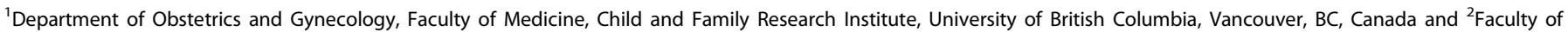
Pharmaceutical Sciences, University of British Columbia, Vancouver, BC, Canada

Correspondence: Dan Rurak (drurak@cw.bc.ca)

Received: 2 May 2018 Revised: 31 October 2018 Accepted: 16 January 2019

Published online: 9 February 2019 
Blood samples were collected following delivery from the ewes via the jugular vein catheter and lambs via jugular venipuncture and also from the lambs on postnatal days (PNDs) 2, 5, 10, and 14 for FX measurement. Electrocardiogram (ECG) measurements were obtained from the lambs for $1 \mathrm{~h}$ on PNDs 2, 5, 10, and 14, using the procedure described below, and tissue $\mathrm{spO}_{2}$ and heart rate were measured using a pulse oximeter (Nonin Medical Inc., Plymouth, MN, USA) clipped to the ear. The values were averaged at 5 min intervals. In addition, the lamb's behavior was assessed using Actiwatch and DVR recordings as described below.

\section{Acute postnatal FX protocol}

Pregnant ewes (Dorset/Suffolk) were transported to the BC Children's Hospital Research Institute at least 3 weeks prior to delivery. Ewes were allowed to deliver spontaneously. The study involved two groups of lambs: a control group $(n=9)$ and one that received bolus intravenous (i.v.) dose of FX $(n=12)$.

Lamb surgeries were conducted on day 2, with anesthesia via $4 \%$ isoflurane and $40 \%$ NO via a face mask and intubation with a cuffed endotracheal tube (3.5-5.0 mm O.D). Anesthesia was maintained with $1.5-2 \%$ isoflurane, $40 \%$ NO in oxygen, and ventilation with an appropriate frequency and tidal volume. ${ }^{8}$ Heparin-bonded 3.5 or 5.0 French polyurethane catheters were implanted into the carotid artery and jugular vein. The catheters were tunneled subcutaneously and exteriorized through a dorsal neck skin incision. Lambs remained in the operating room until conscious and then returned to their mothers.

On PNDs 3 and 4, the lambs were placed in a cloth sling in the pen that contained the lamb's mother. The lambs were suspended above the floor in the sling and normally lay quietly or slept. They were only in the sling for the first $2 \mathrm{~h}$ and then were returned to their mothers, so as not to disrupt the suckling regimen.

Each experiment involved a 30 -min control period followed by i.v. administration of sterile water (day 3) or FX (day $4,1 \mathrm{mg} / \mathrm{kg}$ ) followed by serial collection of arterial blood samples $(\sim 3 \mathrm{ml})$ at $-30,-15,5,15,30,45$, and $60 \mathrm{~min}$ and 2,4, 6, 9, 12, 24, 36, 48, 60, and $72 \mathrm{~h}$. The volume of blood collected represents $\sim 8 \%$ of the total blood volume.

\section{Physiologic data acquisition}

As noted above, in the antenatal FX administration protocol, tissue $\mathrm{spO}_{2}$ and heart rate were measured using a pulse oximeter. In both protocols, the lambs cardiovascular variables were recorded with a polygraph recorder (model TA-4000, Gould Instrument Systems Inc., Valley View, $\mathrm{OH}, \mathrm{USA}$ ) and a Powerlab data acquisition system (AD instrument, Mountain View, CA, USA) This included electroencephalography recordings obtained from three ECG snap electrodes that were attached to the skin on the back and bilaterally on the chest. The raw ECG signal was recorded and in addition this was processed using the Powerlab HRV module to obtain measures of heart rate variability. The Powerlab HRV module measured the beat-to-beat intervals via detection of successive $R$ waves of the ECG signal. Fast Fourier transformation of the R-R intervals allowed estimation of the frequency components: low-frequency (LF) band $(0.04-0.15 \mathrm{~Hz})$ and highfrequency (HF) band $(>0.15 \mathrm{~Hz})$. HRV variables were averaged for 30 -min intervals at $-30,30,60,90$, and $120 \mathrm{~min}$. For the postnatal FX administration, the carotid artery catheter was connected to a disposable pressure transducer (DTX ${ }^{\text {TM }}$ Plus TNF-R; Becton Dickinson, Singapore) to record heart rate and arterial pressure for the first $2 \mathrm{~h}$. Arterial pressure and heart rate were averaged for 1-min intervals for 15 min before FX injection and for 10 min after $\mathrm{FX}$ injection.

Digital video recording and behavioral evaluation

For both protocols, infrared video cameras were installed on the wall of each pen of the holding rooms for continuous video recording and were connected to a computerized digital video recording system. For the prenatal FX exposure protocol, DVR monitoring continued for at least 14 days. For the acute postnatal FX administration protocol, the lamb's activities were monitored for the day of the experiment and the next day. On the day of the acute FX administration, a mobile camera was placed in front of the sling to record the lambs' behavior during the first $2 \mathrm{~h}$ of the experiment. The number of daily sleep and suckling bouts were identified from the DVR observations for each lamb. The total activity was defined as a sum of suckling bouts and active bouts without suckling during each $24 \mathrm{~h}$.

Actiwatch rest/activity recording

In both protocols, an Actiwatch Activity Monitor (AW64, Mini Mitter Inc., Bend, OR, USA) was enclosed in a protective metal case and secured around the neck of the lamb from the first day after birth until at least 14 days after birth to measure rest-activity cycles and sleep patterns. ${ }^{9}$ The activity data was stored in $15 \mathrm{~s}$ epochs and downloaded every 10 days using an Actiwatch reader and analyzed using Actiware-Sleep version 3.5 software (Mini Mitter). The analyzed variables were the same as those collected in a previous study. ${ }^{9}$

Blood sampling and analysis

In the acute postnatal FX protocol, blood samples were serially collected to measure acid-base balance $(\mathrm{pH})$, base excess, bicarbonate $\left(\mathrm{HCO}_{3}{ }^{-}\right)$, arterial blood gases $\left(\mathrm{pO}_{2}, \mathrm{pCO}_{2}\right)$, and electrolyte $\left(\mathrm{Na}^{+}, \mathrm{K}^{+}\right.$, and $\left.\mathrm{iCa}^{2+}\right)$ using an I-STAT-1 analyzer (Abbott, ON, Canada) and $\mathrm{CG}^{+}$cartridges (Abaxis, Union City, (A, USA), FX and and its metabolite norfluoxetine, and cortisol. Body temperature was measured continuously with a thermistor rectal probe (YSI Instruments, Yellow Springs, $\mathrm{OH}, \mathrm{USA}$ ) over the first $2 \mathrm{~h}$ and the blood gas results were corrected for body temperature. Hemoglobin concentration and oxygen saturation were measured with a Radiometer OSM3 Hemoximeter (Radiometer, Copenhagen). Glucose and lactate were determined with a YSI glucose-lactate analyzer (YSI Inc., Yellow Springs, OH, USA).

Drug and hormone assays

Blood samples for hormone and FX measurements were placed in a $4 \mathrm{ml}$ lithium heparin vacutainer tubes (BD Vacutainer, NJ, USA) and centrifuged. Plasma was then transferred to polyvinyl Eppendrorf tubes for hormone samples and glass tubes for FX samples and stored at $-20^{\circ} \mathrm{C}$ until analysis. FX and its metabolite, norfluoxetine, were determined by mass spectrometry (liquid chromatography with tandem mass spectrometry). ${ }^{10}$ Cortisol was measured using a sheep cortisol ELISA kit (Calbiotech Inc.). The sensitivity was $1.38 \mathrm{ng} / \mathrm{ml}$. The intra- and inter-assay coefficients of variation for the low control were $10.7 \%$ and $3.2 \%$, and for the high control were $7.2 \%$ and $6.7 \%$, respectively.

\section{Statistics}

Cardiovascular, blood gas, hormone data, and Actiwatch variables were analyzed using a three-way analysis of variance (ANOVA) followed by Bonferroni's correction for pairwise or control comparisons to determine the effect of treatment, time, and sex. Two-way general linear models for repeated-measures ANOVA was used for the HRV variables, $\mathrm{O}_{2}$ saturation, $\mathrm{pCO}_{2}$, glucose, and hemoglobin $(\mathrm{Hb})$ to determine the effects of treatment and time. Because plasma cortisol concentrations were not normally distributed, the non-parametric Mann-Whitney $U$ test was used. In the antenatal FX exposure experiments, the area under the curve (AUC) was calculated for daily variables (weight, $\mathrm{spO}_{2}$, heart rate, DVR variables) by applying the formula described by Altman. ${ }^{11}$ Then, the AUC of each variable was compared between the control and FX group using the unpaired $t$ test. The effect of FX exposure and sex on gestational age (GA), birth weight, and the time for the lambs to first stand, walk, and suckle were determined using two-way ANOVA. Data are presented as 
TA Nguyen et al.

Table 1. Gestational age and birth weight in the control and antenatally FX-exposed lambs

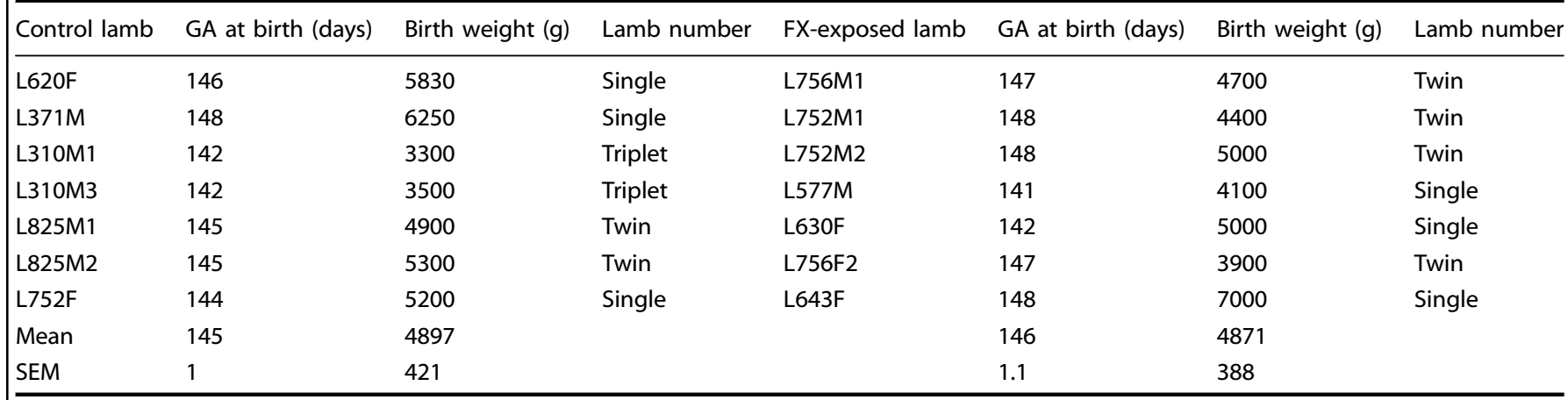

$M$ male, $F$ female. 1, 2, 3 after $\mathrm{M}, \mathrm{F}$ indicates the order of delivery in twin and triplet lambs

$G A$ gestational age, FX fluoxetine

mean \pm SEM. Values were considered to be significantly different with $p<0.05$.

\section{RESULTS}

Prenatal FX exposure protocol

In the FX group, ewes were given FX daily for $12 \pm 1.3$ days. Table 1 describes the GA, birth weight, and sex of the lambs. There were no significant differences. There were also no significant differences in postnatal weight gain between the groups over the first 14 days (data not shown).

At delivery, the mean maternal FX and NFX levels were $627.9 \pm$ 249 and $66.6 \pm 14.4 \mathrm{ng} / \mathrm{ml}$, respectively. The mean plasma FX level in the lambs averaged $17.3 \pm 10.9 \mathrm{ng} / \mathrm{ml}$, decreased progressively thereafter, and became undetectable after day 5 (Fig. 1a). NFX was undetectable in the lambs. The relationship between the lamb/ ewe FX concentration ratio and the duration from the last maternal FX dose is shown in Fig. 1b. The ratio was highest in the animals with the shortest duration from the last FX dose and it fell rapidly thereafter.

There were no differences in the daily tissue $\mathrm{spO}_{2}$ and heart rate values between the control lambs and the FX-exposed lambs (data not shown). $\mathrm{spO}_{2}$ ranged between 90 and $100 \%$ while heart rate decreased progressively in both control and FX lambs from $234 \pm 17$ and $230 \pm 19 \mathrm{bpm}$ on day 2 to $187 \pm 6$ and $200 \pm 8 \mathrm{bpm}$ on day 14 , respectively.

HRV was significantly different on PND 2 (Table 2). The time domain variables of HRV and LF power were significantly lower in the FX-exposed lambs than the control lambs at both 30 and $60 \mathrm{~min}$ of the recording period.

The lambs with prenatal FX exposure were more active than the control lambs, which was reflected by faster first time activities (Table 3) and an increase in subsequent activities obtained from Actiwatch and DRV observations (Figs. 2, 3). Figure 2 shows the Actiwatch variables from PNDs 2 to 14 in control and FX-exposed lambs. The lambs' activity increased with time in both groups, but it was increasingly greater in the FX group. Total activity score (Fig. 2a) and active bouts per day (Fig. 2b) were significantly higher in the FX-exposed group, while they were comparable between the two groups on PNDs 2 to 3. Mean score in active periods (Fig. 2c) was significantly higher. Episode duration (Fig. 2d) was significantly lower in the FX group on PNDs 5, 9, 10, and 12. Thus, the activity with prenatal FX exposure was more disrupted, in terms of more active, but shorter active bouts/day. The percentage of time moving (Fig. 2e) was significantly higher in the FX group on PNDs 2 to 12, with a reverse situation for the percentage of time immobile (Fig. 2f).

From the DVR observations, the FX-exposed lambs had a significantly lower number of suckling bouts on PNDs 2, 5, and 6
(Fig. 3b). However, the AUC of non-suckling activity bouts for the first 10 PNDs was significantly higher in the FX-exposed lambs (Fig. 3c). Therefore, the number of total activity bouts (=suckling bouts + non-suckling active bouts) was comparable between the two groups until PND 9 (Fig. 3d) and so was the number of sleep bouts (Fig. 3a). The non-suckling behavior in the FX-exposed lambs largely comprised standing still. On PND 10, the number of total activity bouts was significantly lower in the FX group and so were sleep bouts, but there were no significant differences in the number of suckling bouts. In addition, we did not observe any other abnormal behaviors in the FX-exposed lambs.

Acute postnatal FX exposure protocol

On days $3.3 \pm 0.4$ and $4.2 \pm 0.4$ sterile water $(0.1 \mathrm{ml} / \mathrm{kg}, N=9)$ or $\mathrm{FX}$ ( $1 \mathrm{mg} / \mathrm{kg}, N=12$ ) were acutely injected. There were five male and seven females in the FX group and there were no sex differences in terms of the drug effects.

Plasma FX concentration and weight gain

The plasma FX concentrations over the experiment and in 13 individual human infants sampled at PND 2 following in utero exposure to the drug ${ }^{12}$ cover a similar range $(\sim 10-100 \mathrm{ng} / \mathrm{ml})$ (Fig. 4). Norfluoxetine was not detected. There were also no significant differences in postnatal weight gain on PND 4 between the control group $(0.411 \pm 0.056 \mathrm{~kg}, n=9)$, FX group $(0.392 \pm$ $0.045 \mathrm{~kg}, n=12$ ) and the FX group on the day after FX administration $(0.329 \pm 0.043 \mathrm{~kg})$.

Rest-activity cycles

The Actiwatch variables were comparable between the control group with sterile water injection $(n=9)$ and the FX group $(n=$ 12). (data not shown) In addition, from the DVR observation there were no obvious behavioral changes following FX dosing; the lambs just lay quiet or slept for most of the time.

Cardiovascular variables (heart rate, arterial pressure, HRV) Arterial pressure was significantly different between the control and FX group at 1, 2, 3, 4, 5, and 10 min post FX (Fig. 5a). The maximum increase in arterial pressure in the FX group was $8.5 \pm$ $1.1 \mathrm{mmHg}$ at $4 \mathrm{~min}$ following FX. Heart rate was lower by $38 \pm$ $9 \mathrm{bpm}$ in the FX group than the control group only at 2 min post FX (Fig. 5b).

The time domain of HRV, which are related to the R-R interval variability, increased after injection (Fig. 6 a-c). In contrast, the frequency domain HRV variables, including LF power, HF power, and LF/HF ratio, which indicate cardiac parasympathetic and sympathetic influences, were not changed (Fig. $6 \mathrm{~d}-\mathrm{f}$ ). There was a significant inverse relationship between the fall in heart rate and the increase in SDNN (standard deviation of NN (normal-to-normal 
a
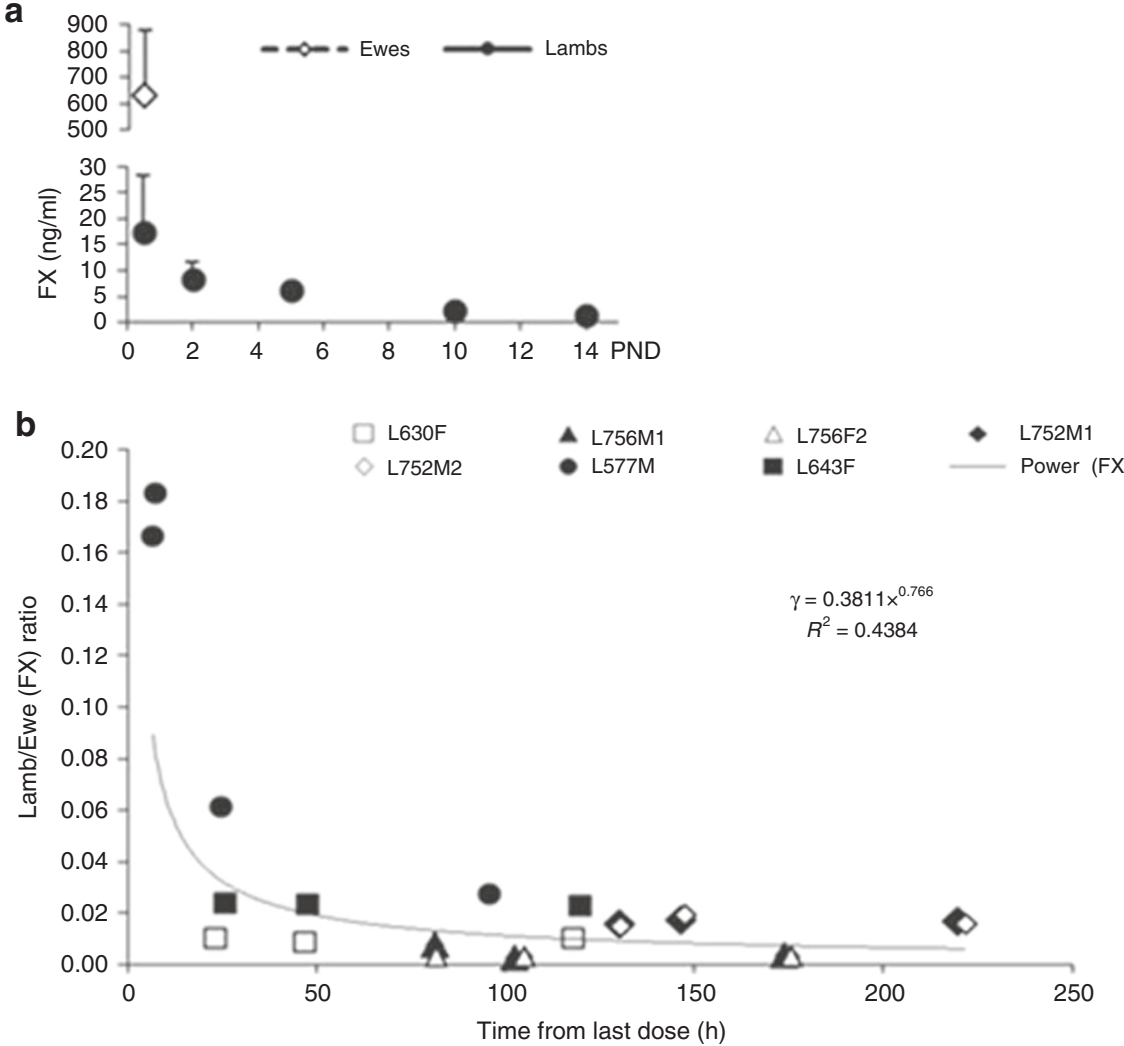

Fig. 1 a Fluoxetine (FX) concentrations in the prenatally FX-exposed ewes $(n=5)$ and lambs $(n=7)$. PND $=$ postnatal day. $\mathbf{b}$ The relationship between the lamb:ewe plasma FX concentration ratio and the time from the last maternal FX dose

Table 2. Heart rate variability variables (time domain: SDNN, SD Delta NN, RMSSD; frequency domain: total power, LF power, HF power, LF/ $\mathrm{HF}$ ) on postnatal day 2 in the control group $(n=8)$ and the prenatal FX-exposed lambs $(n=7)$

\begin{tabular}{lcc}
\hline Variable & Control group & FX-exposed group \\
\hline Heart rate (bpm) & $220 \pm 4.3$ & $225 \pm 6.0$ \\
SDNN (ms) & $22.5 \pm 2.3$ & $15.5 \pm 0.8^{*}$ \\
SD Delta NN & $18.8 \pm 4.8$ & $7.2 \pm 4.4^{*}$ \\
RMSSD & $18.4 \pm 4.8$ & $7.2 \pm 4.8^{*}$ \\
LF power $\left(\mathrm{ms}^{2}\right)$ & $73.8 \pm 11.5$ & $37.5 \pm 4.7^{*}$ \\
HF power $\left(\mathrm{ms}^{2}\right)$ & $51.4 \pm 13.4$ & $19.2 \pm 4.8$ \\
LF/HF & $2.2 \pm 0.6$ & $2.6 \pm 0.5$ \\
\hline SDNN standard deviation of NN (normal-to-normal intervals), SD Delta NN \\
standard deviation of differences in the NN interval, RMSSD square root of \\
the mean squared difference of successive NN intervals, LF low frequency, \\
$\begin{array}{l}\text { HF high frequency, FX fluoxetine } \\
\text { *significantly different from the corresponding control value }\end{array}$ \\
\hline
\end{tabular}

Table 3. First time activities in control newborn lambs and newborn lamb exposed antenatally to FX

\begin{tabular}{llll}
\hline Group $(n)$ & $\begin{array}{l}\text { Time to stand } \\
(\mathrm{min})\end{array}$ & $\begin{array}{l}\text { Time to walk } \\
(\mathrm{min})\end{array}$ & $\begin{array}{l}\text { Time to suckle } \\
(\mathrm{min})\end{array}$ \\
\hline Control (7) & $32.2 \pm 2$ & $45.0 \pm 4$ & $56.0 \pm 5$ \\
FX (7) & $17.0 \pm 3^{*}$ & $26.3 \pm 4^{*}$ & $29.0 \pm 6^{*}$ \\
\hline $\begin{array}{l}\text { FX fluoxetine } \\
\text { *Significantly different from the control value }\end{array}$ \\
\hline
\end{tabular}

intervals)) (Supplemental Fig. 1), with similar relationships with SD delta NN (standard deviation of differences in the NN interval) and RMSSD (square root of the mean squared difference of successive NN intervals) (data not shown).

Arterial blood gases

The mean values for arterial $\mathrm{pO}_{2}, \mathrm{pCO}_{2}, \mathrm{pH}$, base excess, $\mathrm{Hb}$ concentration, $\mathrm{O}_{2}$ saturation, and glucose and lactate concentrations were $87.1 \pm 3.3 \mathrm{mmHg}, 40.3 \pm 1.0 \mathrm{mmHg}, 7.439 \pm 0.019,3.44$ $\pm 1.41,12.4 \pm 0.34 \mathrm{~g} / \mathrm{l}, 94.3 \pm 0.4 \%, 5.5 \pm 0.23 \mathrm{~mm} / \mathrm{l}, 1.03 \pm 0.08 \mathrm{~mm}$ in the control lambs and $88.0 \pm 3.8 \mathrm{mmHg}, 42.2 \pm 1.0 \mathrm{mmHg}$, $7.385 \pm 0.010,0.27 \pm 0.75,11.2 \pm 0.5 \mathrm{~g} \%, 93.3 . \pm 0.4 \%, 5.49 \pm 0.25$ $\mathrm{mM}$ and $0.90 \pm 0.05 \mathrm{~mm}$ in the FX group. There were no differences between the groups and no changes with time.(data not shown).

Plasma cortisol concentration

No differences were found between the cortisol concentrations in the control group and FX group. In both groups, the cortisol level was significantly higher at $30 \mathrm{~min}, 1 \mathrm{~h}$, and $2 \mathrm{~h}$ than that at $12 \mathrm{~h}$ following FX injection (Supplemental Fig. 2).

\section{DISCUSSION}

Prenatal FX exposure protocol

The maternal FX level at delivery was higher than that reported in the human ${ }^{12}$ and in our previous sheep studies. ${ }^{13}$ CYP2D6 is a key enzyme involved in FX metabolism. ${ }^{14}$ Moreover, FX isomers are potent inhibitors of CYP2D6, ${ }^{15}$ so that the elevated maternal FX might be due to FX-mediated CYP2D6 inhibition. ${ }^{15}$ One case report gave a neonatal FX concentration at delivery of $26 \mathrm{ng} / \mathrm{ml}$ and undetectable levels at $96 \mathrm{~h}$ thereafter, ${ }^{16}$ which is similar to the current results. The FX level in our lambs at birth $(17.3 \pm 10.9 \mathrm{ng} / \mathrm{ml})$ 

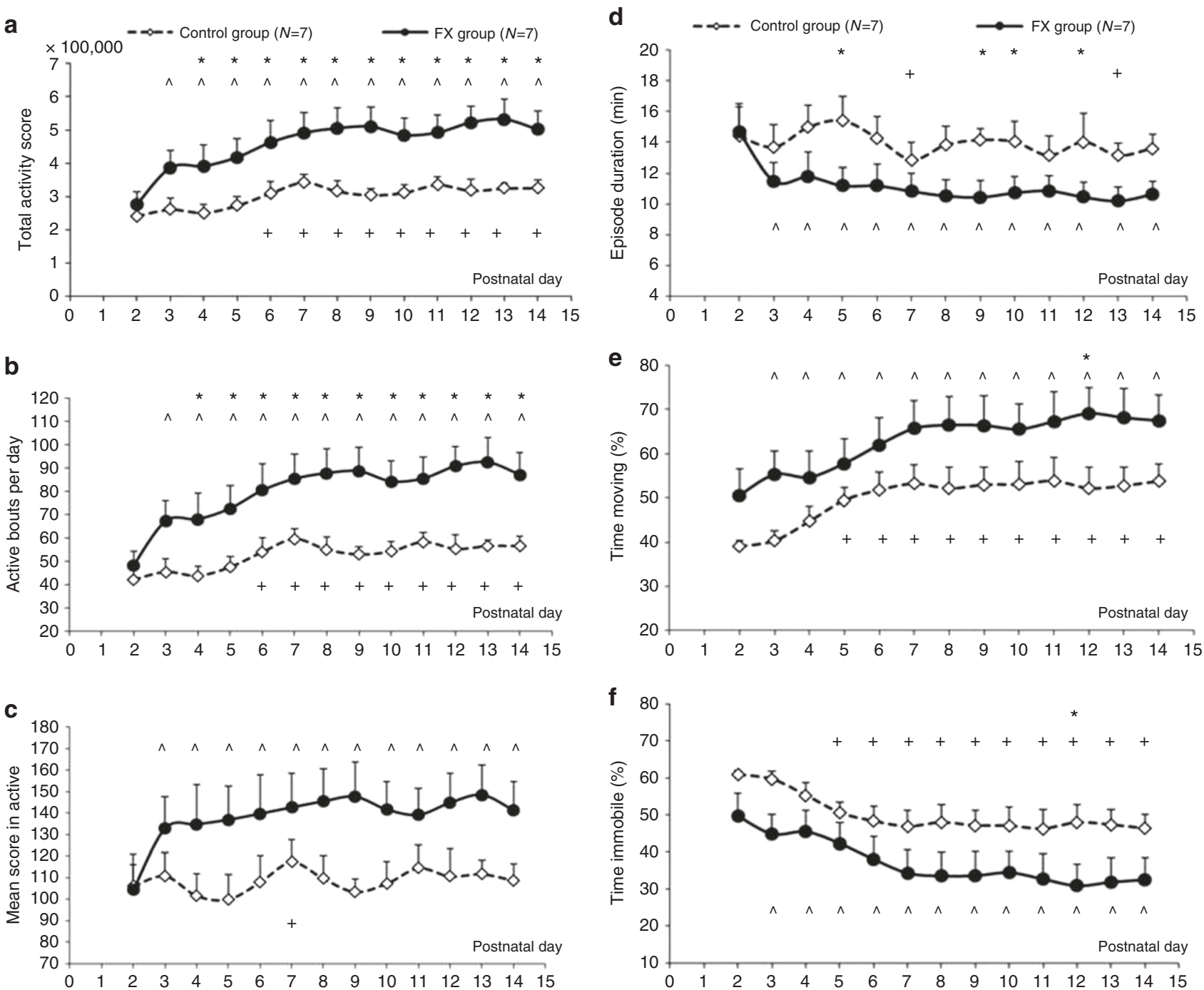

Fig. 2 Daily activity variables obtained from the Actiwatches in the control (open, diamond symbols) and prenatal fluoxetine (FX)-exposed lambs (closed, circle symbols). ${ }^{*}$ indicates significant differences in the corresponding values between the control and FX group; + indicates significant difference from the day 2 within the control group; $\wedge$ indicates significant difference from the day 2 within the FX group

was lower than that measured in the umbilical cord vein at delivery $(41.3 \mathrm{ng} / \mathrm{ml})$, from pregnant women on FX therapy. ${ }^{12}$ Thus, in this study, one might expect to see a higher level of FX in the lambs than was actually the case. However, there is also a relationship between the ratio of lamb/ewe FX concentration at delivery and the duration from the maternal last FX dose (Fig. 1b). This suggests that following the last maternal FX injection, the elimination of FX from the fetus was largely the result of transfer of the drug back to the mother and maternal elimination. NFX was undetectable at birth and afterwards, which suggests that fetal and newborn lambs are unable to metabolize the drug, similar to findings in the fetal lamb. ${ }^{13}$

There were no differences in birth weight or gestation length between the experimental and control groups (Table 1), although these results may be have affected by the inclusion of twins and triplets. However, we have previously reported that lamb number has no effect on gestation length in sheep, while birth weight is decreased progressively with increasing lamb number. ${ }^{18}$ This latter phenomenon is evident in Table 1, but the similar distribution of twins and triplets in the two groups likely minimized the effect on mean birth weights.

The significantly lower time domain variables of HRV and total power in the prenatally FX-exposed lambs on PND 2 is opposite from the acute FX injection results where these variables increased transiently (Fig. 6). Prenatal exposure to SSRIs reduces short- and long-term HRVs at 36 weeks gestation. ${ }^{19}$ It seems possible that in the current study of prenatal FX exposure, fetal HRV was reduced in utero, which persisted until day 2 when FX was still present. The current and published results suggest that the reduced postnatal HRV may originate in the fetal period associated with SSRI exposure, but further research is needed.

Lambs that are slow to stand and suckle after birth have a lower survival chance. ${ }^{20}$ Our finding that FX-exposed lambs were faster to stand, walk and suckle as compared to the control lambs is different from a human study in which the times to first sit and walk were delayed in infants exposed to antidepressants during the second and third trimesters. ${ }^{21}$ However, in this study, different classes of antidepressants were included and the duration of drug exposure was much greater.

For the first 2 weeks of life, there was a progressive increase in the level of lambs' activity in both the FX-exposed and control groups, which is similar to previous results., ${ }^{9,22}$ Although the FXexposed lambs were more active, it appeared that their movement bouts were shorter, which was reflected by significantly lower values for episode duration in the FX group on PNDs 5, 9, 10, and 12. Similar to our findings, a study in human infants found 

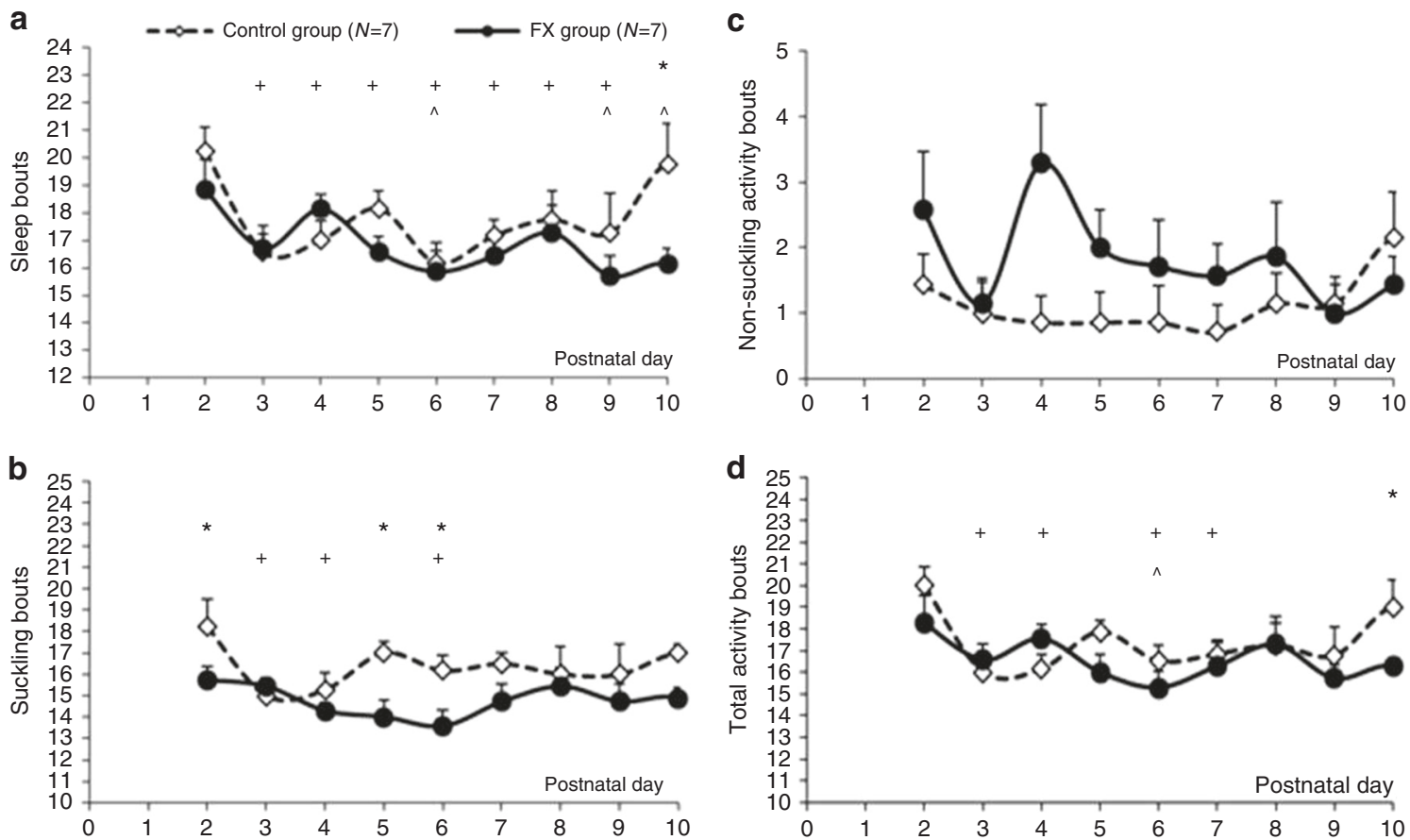

Fig. 3 Daily sleep, suckling, and non-suckling activity bouts obtained from digital video recorder (DVR) observations in the control (open, diamond symbols) and prenatal fluoxetine (FX)-exposed lambs (closed, circle symbols). ${ }^{*}$ indicates significant differences in the corresponding values between the control and FX. ${ }^{+}$indicates significant differences from the day 2 within the control group. $\wedge$ indicates significant differences from the day 2 within the FX group

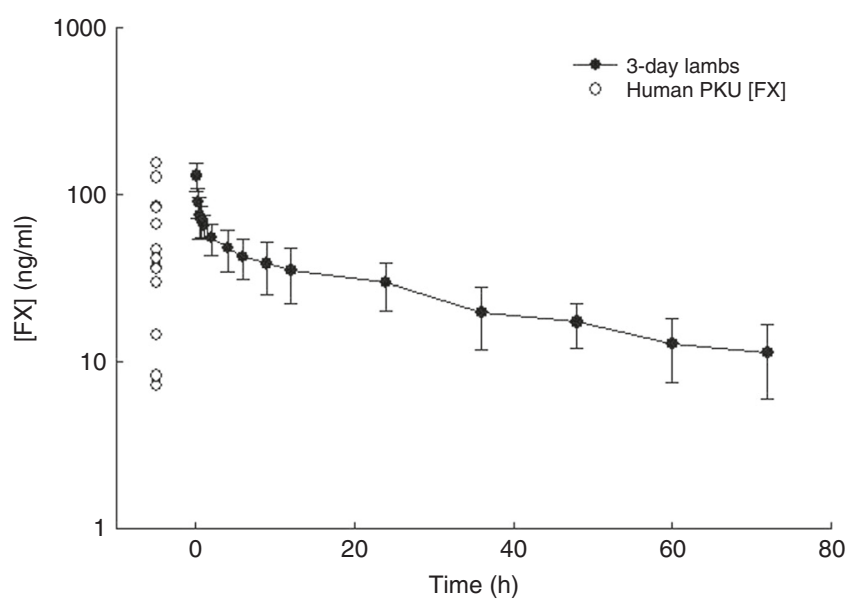

Fig. 4 Plasma fluoxetine (FX) concentration versus time in 4-day-old lambs (closed symbols and solid line) and in 13 individual human infants (open symbols) sampled at postnatal day 2 following in utero FX exposure ${ }^{12}$

prenatally SSRI-exposed infants increase their motor activity during sleep compared to controls; ${ }^{23}$ however, the difference in activities between the groups was attributed to the shorter gestational age in the SSRI-exposed infants, which was not the case here. Lambs are more active during the first month of life than later and this is due to their frequent suckling bouts. ${ }^{22}$ However, the DVR observations revealed that the increase in the activity level in FX-exposed lambs was not due to increased suckling, which is the major activity in normal lambs in the first month of life. ${ }^{22}$ Instead, the increased activity level was due to the higher number of non-suckling bouts and these were largely comprised of standing still. This is consistent with the lack of a

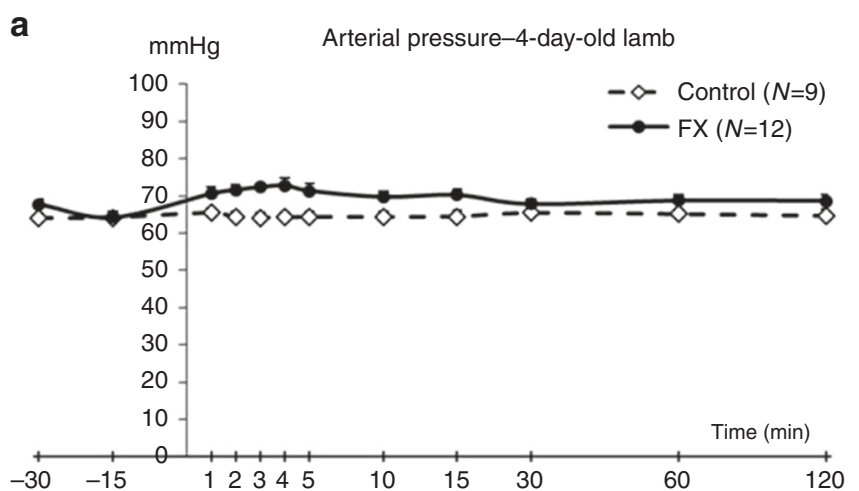

b

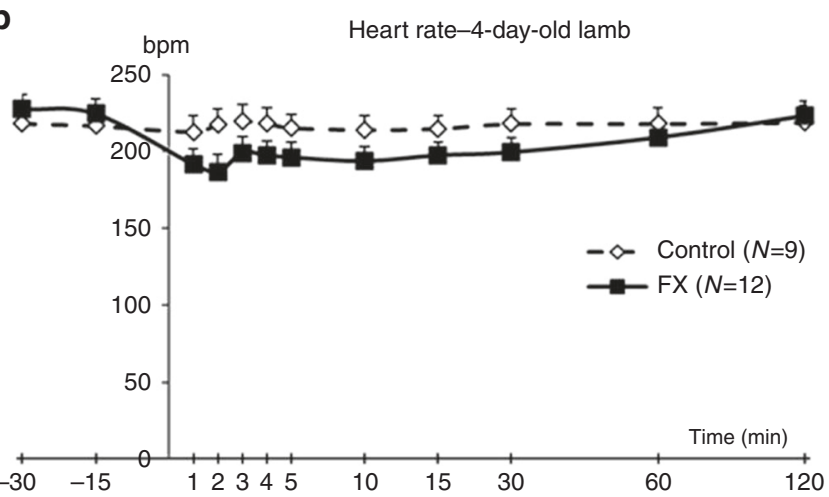

Fig. 5 Arterial pressure and heart rate in 4-day-old lambs in the control and fluoxetine (FX) groups. a indicates significant differences between variables compared to $-15 \mathrm{~min}$; $\mathbf{b}$ indicates significant differences between control and FX groups 

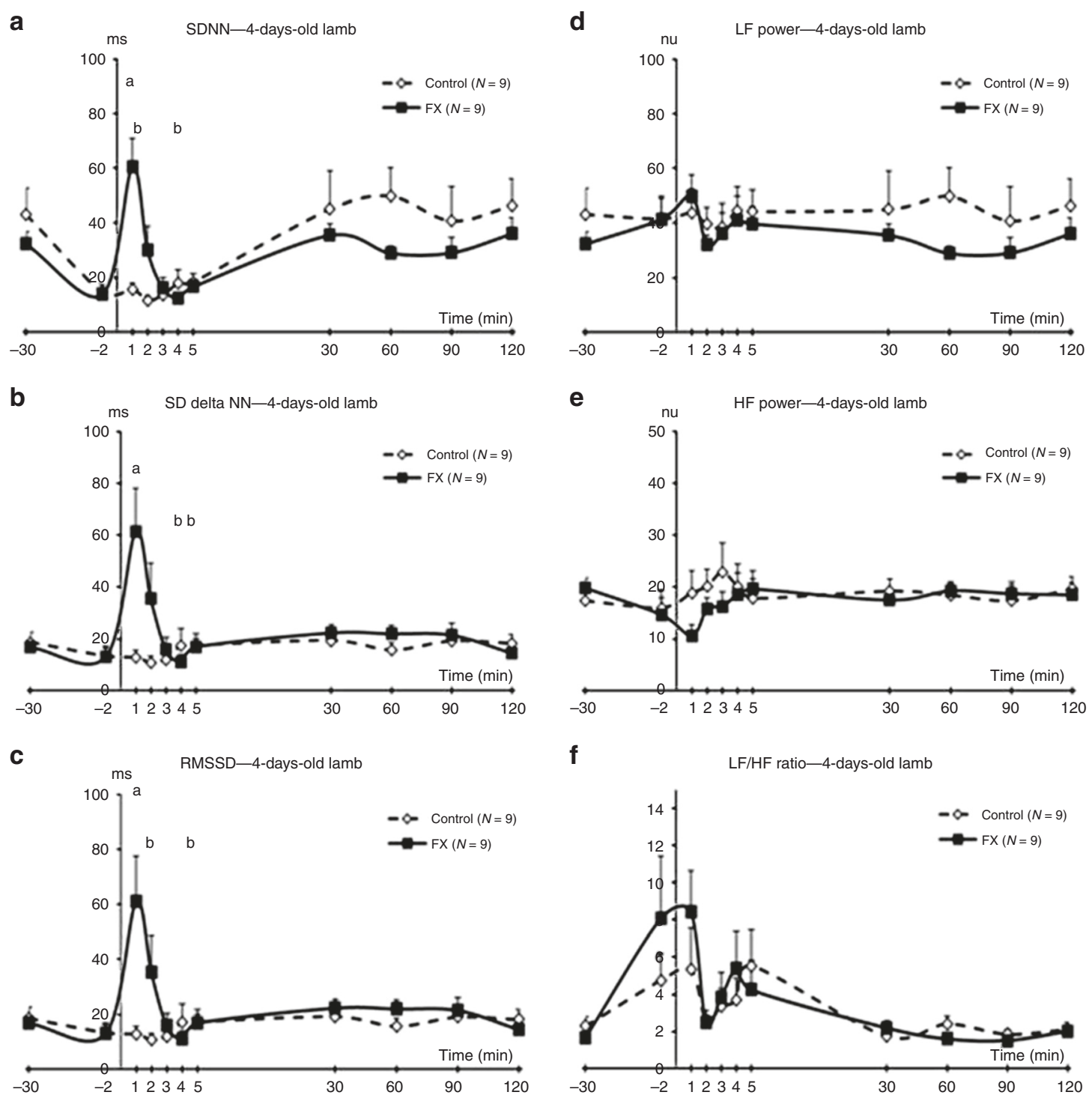

Fig. 6 Time and frequency domains of heart rate variability (HRV) in the control group (open diamond and dotted line) and fluoxetine (FX) group (closed square and solid line). SDNN, standard deviation of NN (normal-to-normal intervals), SD Delta NN, standard deviation of differences in the NN interval, RMSSD, square root of the mean squared difference of successive NN intervals; LF and HF power and the LF/HF ratio are determined using power spectral analysis of HRV, LF power, low-frequency power, HF power, high-frequency power, LF/HF, the ratio between LF and HF power. a indicates significant differences between variables compared to the pre-FX value; $b$ indicates significant differences between the control and FX groups. (a) SDNN (b) SD Deta NN (c) RMSSD (d) LF Power (e) HF Power (f) LF/HF

significant difference in postnatal weight gain between the two groups. The increase in activity in the FX-exposed lambs is not seen in normal lambs, which over the first 2 postnatal weeks spend the bulk of their time either sleeping or suckling. ${ }^{22}$

The behavioral differences in the FX-exposed lambs could represent increased maturation of locomotory activities, which could be of survival advantage to the animals. We have found that in normal lambs, total activity recorded by actigraphy increases progressively until about 15 days following birth, ${ }^{22}$ and this appears related to maturation of muscle and bone tissue after birth. ${ }^{9}$ Thus, the increased activity in the FX-exposed lambs suggests that they were more mature at birth in terms of bone and muscle development and those aspects of brain development that controls voluntary movements. However, in normal lambs the increase in activity over the first 15 PNDs largely results from changes in suckling bouts and suckling duration. ${ }^{22}$ Given that greater maturity of lambs than human newborns in terms of locomotory activity, the hyperactivity in the FX-exposed lambs may be comparable to the jitteriness, one of the symptoms of poor neonatal adaptation. ${ }^{3}$

In addition, from the DVR observations, on PND 10, FX-exposed lambs had a significantly lower number of sleep bouts, suggesting that they slept for a longer duration. However, on this day, FXexposed lambs had a shorter sleep episode duration from the Actiwatch measurements. This suggests that sleep in the FXexposed lambs was more disrupted than the control, which was also the case with the active bouts.

Acute postnatal FX exposure cohort study

Previous studies have reported that antenatally SSRI-exposed infants are at increased risk of being growth restricted ${ }^{2}$ and having reduced postnatal weight gain. ${ }^{24}$ This could be due to the higher incidence of premature birth in the SSRI group, ${ }^{2}$ or to reduced fetal oxygen and nutrient supply via a reduction in uterine blood flow due to an increased plasma serotonin level ${ }^{25}$ or to a direct effect of serotonin on skeleton and bone growth. ${ }^{26}$ Similarly, there 
Postnatal outcomes in lambs exposed antenatally and acutely postnatally... TA Nguyen et al.

is reduced postnatal weight gain in lambs exposed antenatally to $\mathrm{FX}^{25}$ However, in this study, the postnatal weight gain was comparable between the experimental and control groups. The active bouts per day were also not different between the two groups. Thus, the decreased postnatal weight gain reported in antenatally FX-exposed human infants and in postnatal lambs exposed in FX in late gestation does not appear due to the persistence of the drug in the newborns, given that the plasma FX concentrations in the lambs are within the range of that observed in human infants on PND 2 following antenatal FX exposure. ${ }^{12}$

SSRIs cause an acute increase in plasma serotonin levels through inhibiting serotonin reuptake by platelets. In humans, mice, and sheep, acute FX administration increases plasma serotonin concentration. ${ }^{25,27-29}$ Serotonin is a vasoconstrictor and in sheep causes dose-related increases in blood pressure. ${ }^{29}$ This agrees with our findings that arterial pressure increased at 2-10 min after FX administration. Heart rate also declined at 2 min post-FX. However, these effects were very transient and were not of any clinical significance.

The transient bradycardia could be due to either a direct effect of $\mathrm{FX}$ on the cardiomyocytes via $5-\mathrm{HT}_{4}$ receptors or indirectly via a baroreflex response to the hypertension. ${ }^{30}$ Following FX injection, there were statistically significant, transient changes in HRV time domain variables, associated with the bradycardia, and there was a statistically significant inverse relationship between heart rate and SDNN. We have found a similar relationship between heart rate and these HRV variables in lambs between birth and 1 year of age $^{22}$ and similar findings have been reported in the human fetus, infant, and child. ${ }^{31-33}$

As noted previously, FX i.v. infusion in pregnant ewes resulted in an augmented prepartum rise in fetal cortisol levels. ${ }^{7}$ In contrast, in utero SSRI exposure is associated with reduced basal cortisol levels and an alteration in the HPA stress response in 3-month-old infants. ${ }^{34}$ There is also a lower cord blood cortisol level in SSRIexposed neonates. ${ }^{26}$ These results are different from those obtained in the antenatal FX administration protocol, where the drug had no effect on plasma cortisol. These conflicting findings could be due to differences in the timing and duration of SSRI exposure. Acute administration of SSRIs increases the secretion of cortisol and $\mathrm{ACTH}^{35}$ while chronic treatment with citalopram results in a decrease. ${ }^{36}$

The mechanisms of poor neonatal adaptation in newborns exposed in utero to SSRIs are still a matter of debate. It is apparent that the high level of activity in our antenatally FX-exposed lambs was not due to FX toxicity since acute FX administration on PND 4 resulted in no behavioral effects. It also seems unlikely that it is due to drug withdrawal, since the high level of activity in the FXexposed lambs persisted at least until PND 14, days after detectable FX. In addition, there is the abnormal phenomenon of increased body movements during non-REM sleep in SSRIexposed human fetuses. ${ }^{37}$ Similarly, another study found SSRIexposed fetuses had a longer umbilical cord than control fetuses, suggesting that the former group were more active. ${ }^{38}$ And as noted previously, we reported that FX administration to late gestation pregnant sheep led to a persistent decrease in fetal REM sleep. ${ }^{7}$ Collectively, these findings support the hypothesis that a possible underlying mechanism for the high level of activity in the FX-exposed lambs is SSRI-induced changes in fetal brain development, particularly since both sleep and active periods appeared disrupted. Further evidence for this mechanism comes from studies showing a lower cord blood level of calcium-binding protein S100B, a biomarker of early brain development and central serotonergic function, in SSRI-exposed neonates. ${ }^{39}$ Overall these results suggest that poor neonatal adaptation associated with prenatal SSRI exposure is not due to SSRI toxicity or a withdrawal effect. Rather, it may result from altered fetal brain development with SSI exposure.

\section{ACKNOWLEDGEMENTS}

This research was funded by the Canadian Institutes of Health Research (MOP-84477) TAN was a recipient of IWRH and CFRI studentship award.

\section{ADDITIONAL INFORMATION}

The online version of this article (https://doi.org/10.1038/s41390-019-0309-8) contains supplementary material, which is available to authorized users.

Competing interests: The authors declare no competing interests.

Publisher's note: Springer Nature remains neutral with regard to jurisdictional claims in published maps and institutional affiliations.

\section{REFERENCES}

1. Andrade, S. E. et al. Use of antidepressant medications during pregnancy: a multisite study. Am. J. Obstet. Gynecol. 198, 194.e1-194.e5 (2008).

2. Oberlander, T. F., Warburton, W., Misri, S., Aghajanian, J. \& Hertzman, C. Neonatal outcomes after prenatal exposure to selective serotonin reuptake inhibitor antidepressants and maternal depression using population-based linked health data. Arch. Gen. Psychiatry 63, 898-906 (2006).

3. Moses-Kolko, E. L. et al. Neonatal signs after late in utero exposure to serotonin reuptake inhibitors: literature review and implications for clinical applications. JAMA 293, 2372-2383 (2005)

4. Kieviet, N. et al. Serotonin and poor neonatal adaptation after antidepressant exposure in utero. Acta Neuropsychiatr. 29, 43-53 (2017).

5. Levinson-Castiel, R., Merlob, P., Linder, N., Sirota, L. \& Klinger, G. Neonatal abstinence syndrome after in utero exposure to selective serotonin reuptake inhibitors in term infants. Arch. Pediatr. Adolesc. Med. 160, 173-176 (2006).

6. Kieviet, N. et al. Risk factors for poor neonatal adaptation after exposure to antidepressants in utero. Acta Paediatr. 104, 384-391 (2015).

7. Morrison, J. L., Riggs, K. W. \& Rurak, D. W. Fluoxetine during pregnancy: impact on fetal development. Reprod. Fertil. Dev. 17, 641-650 (2005).

8. Moss, T. J., Jakubowska, A. E., McCrabb, G. J., Billings, K. \& Harding, R. Ventilatory responses to progressive hypoxia and hypercapnia in developing sheep. Respir. Physiol. 100, 33-44 (1995).

9. Rurak, D. W., Fay, S. \& Gruber, N. C. Measurement of rest and activity in newborn lambs using actigraphy: studies in term and preterm lambs. Reprod. Fertil. Dev. 20, 418-430 (2008).

10. Chow, T. W., Szeitz, A., Rurak, D. W. \& Riggs, K. W. A validated enantioselective assay for the simultaneous quantitation of $(R)$-, (S)-fluoxetine and $(R)$-, $(S)$-norfluoxetine in ovine plasma using liquid chromatography with tandem mass spectrometry (LC/MS/MS). J. Chromatogr. B 879, 349-358 (2011).

11. Altman, D. Some common problems in medical research. In: Practical Statistics for Medical Research. London: Chapman \& Hall. (pp. 426-439). (1991).

12. Kim, J. et al. Stereoselective disposition of fluoxetine and norfluoxetine during pregnancy and breast-feeding. Br. J. Clin. Pharmacol. 61, 155-163 (2006).

13. Kim, J., Riggs, K. W. \& Rurak, D. W. Stereoselective pharmacokinetics of fluoxetine and norfluoxetine enantiomers in pregnant sheep. Drug Metab. Dispos. 32 212-221 (2004).

14. Ring, B. J. et al. Identification of the human cytochromes p450 responsible for in vitro formation of $R$ - and S-norfluoxetine. J. Pharmacol. Exp. Ther. 297, 1044-1050 (2001).

15. Alfaro, C. L., Lam, Y. W., Simpson, J. \& Ereshefsky, L. CYP2D6 inhibition by fluoxetine, paroxetine, sertraline, and venlafaxine in a crossover study: intraindividual variability and plasma concentration correlations. J. Clin. Pharmacol. 40, 58-66 (2000).

16. Rampono, J., Proud, S., Hackett, L. P., Kristensen, J. H. \& Ilett, K. F. A pilot study of newer antidepressant concentrations in cord and maternal serum and possible effects in the neonate. Int. J. Neuropsychopharmacol. 7, 329-334 (2004).

17. Spencer, M. J. Fluoxetine hydrochloride (Prozac) toxicity in a neonate. Pediatrics 92, 721-722 (1993)

18. Bessette, N. W. \& Rurak, D. W. Chronic fetal and maternal instrumentation in pregnant sheep: effect on gestation length and birthweight. Reprod. Fertil. Dev. 22, 459-467 (2010).

19. Rurak, D. et al. Third trimester fetal heart rate and Doppler middle cerebral artery blood flow velocity characteristics during prenatal selective serotonin reuptake inhibitor exposure. Pediatr. Res. 70, 96-101 (2011).

20. Dwyer, C. M., Calvert, S. K., Farish, M., Donbavand, J. \& Pickup, H. E. Breed, litter and parity effects on placental weight and placentome number, and consequences for the neonatal behaviour of the lamb. Theriogenology 63, 1092-1110 (2005). 
21. Pedersen, L. H., Henriksen, T. B. \& Olsen, J. Fetal exposure to antidepressants and normal milestone development at 6 and 19 months of age. Pediatrics 125 , e600-e608 (2010).

22. Nguyen, T. T. Cardiovascular, metabolic, endocrine and behavioral aspects of development in postnatal lambs in relation to acute fluoxetine administration. PhD Thesis, University of British Columbia (https://open.library.ubc.ca/clRcle/ collections/ubctheses/24/items/1.0073940) (2013).

23. Zeskind, P. S. \& Stephens, L. E. Maternal selective serotonin reuptake inhibitor use during pregnancy and newborn neurobehavior. Pediatrics 113, 368-375 (2004).

24. Chambers, C. D. et al. Weight gain in infants breastfed by mothers who take fluoxetine. Pediatrics 104, e61 (1999).

25. Morrison, J. L., Chien, C., Riggs, K. W., Gruber, N. \& Rurak, D. Effect of maternal fluoxetine administration on uterine blood flow, fetal blood gas status and growth. Pedia. Res 51, 433-442 (2002).

26. Davidson, S. et al. Effect of exposure to selective serotonin reuptake inhibitors in utero on fetal growth: potential role for the IGF-I and HPA axes. Pediatr. Res. $\mathbf{6 5}$, 236-241 (2009).

27. Ortiz, J. \& Artigas, F. Effects of monoamine uptake inhibitors on extracellular and platelet 5-hydroxytryptamine in rat blood: different effects of clomipramine and fluoxetine. Br. J. Pharmacol. 105, 941-946 (1992).

28. Maurer-Spurej, E., Pittendreigh, C. \& Solomons, K. The influence of selective serotonin reuptake inhibitors on human platelet serotonin. Thromb. Haemost. 91, 119-128 (2004)

29. Alvarez, J. C. et al. Decreased platelet serotonin transporter sites and increased platelet inositol triphosphate levels in patients with unipolar depression: effects of clomipramine and fluoxetine. Clin. Pharmacol. Ther. 66, 617-624 (1999).
30. Nelson, M. et al. Ritanserin and serotonergic mechanisms in blood pressure and fluid regulation in sheep. Clin. Exp. Pharmacol. Physiol. 14, 555-563 (1987).

31. Watts, S. W. \& Davis, R. P. 5-Hydroxtryptamine receptors in systemic hypertension: an arterial focus. Cardiovasc. Ther. 29, 54-67 (2011)

32. Lange, S., Van Leeuwen, P., Geue, D., Hatzmann, W. \& Gronemeyer, D. Influence of gestational age, heart rate, gender and time of day on fetal heart rate variability. Med. Biol. Eng. Comput. 43, 481-486 (2005).

33. Massin, M. \& von Bernuth, G. Normal ranges of heart rate variability during infancy and childhood. Pediatr. Cardiol. 18, 297-302 (1997).

34. Oberlander, T. F. et al. Hypothalamic-pituitary-adrenal (HPA) axis function in 3month old infants with prenatal selective serotonin reuptake inhibitor (SSRI) antidepressant exposure. Early Hum. Dev. 84, 689-697 (2008).

35. Raap, D. K., Van \& de Kar, L. D. Selective serotonin reuptake inhibitors and neuroendocrine function. Life. Sci. 65, 1217-1235 (1999).

36. Jongsma, M. E., Bosker, F. J., Cremers, T. I., Westerink, B. H. \& den Boer, J. A. The effect of chronic selective serotonin reuptake inhibitor treatment on serotonin $1 B$ receptor sensitivity and HPA axis activity. Prog. Neuropsychopharmacol. Biol. Psychiatry 29, 738-744 (2005).

37. Mulder, E. J., Ververs, F. F., de Heus, R. \& Visser, G. H. Selective serotonin reuptake inhibitors affect neurobehavioral development in the human fetus. Neuropsychopharmacology 36, 1961-1971 (2011).

38. Kivisto, J., Lehto, S. M., Halonen, K., Georgiadis, L. \& Heinonen, S. Maternal use of selective serotonin reuptake inhibitors and lengthening of the umbilical cord: indirect evidence of increased foetal activity - a retrospective cohort study. PLoS ONE 11, e0154628 (2016).

39. Pawluski, J. L., Galea, L. A., Brain, U., Papsdorf, M. \& Oberlander, T. F. Neonatal S100B protein levels after prenatal exposure to selective serotonin reuptake inhibitors. Pediatrics 124, e662-e670 (2009). 